\title{
Anestesia local por tumescência com lidocaína em cadelas submetidas a mastectomia
}

[Tumescent local anesthesia with lidocaine in dogs undergoing mastectomy]

\author{
C.J.X. Abimussi ${ }^{1}$, J.Z. Ferreira ${ }^{2}$, B.P. Floriano ${ }^{2}$, F. Paes ${ }^{3}$, S.H.V. Perri ${ }^{4}$, V.N.L.S. Oliva ${ }^{4}$
}

\author{
${ }^{1}$ Aluno de pós-graduação - Faculdade de Medicina de Botucatu (FMB-Unesp) - Botucatu, SP \\ ${ }^{2}$ Aluna de pós-graduação - Faculdade de Medicina Veterinária de Araçatuba (FMVA-Unesp) - Araçatuba, SP \\ ${ }^{3}$ Programa de Residência - Faculdade de Medicina Veterinária de Araçatuba (FMVA-Unesp) - Araçatuba, SP \\ ${ }^{4}$ Faculdade de Medicina Veterinária de Araçatuba (FMVA-Unesp) - Araçatuba, SP
}

\begin{abstract}
RESUMO
O presente estudo investigou os benefícios da anestesia por tumescência com lidocaína em cadelas submetidas à mastectomia, visando ao conforto do paciente e à sua recuperação pós-operatória. Foram utilizados sete animais, de peso e raças variadas, que apresentavam neoplasia em região de cadeia mamária e que foram submetidos à cirurgia de mastectomia. Todos os animais receberam o mesmo protocolo anestésico, sendo utilizado como MPA a associação entre acepromazina e morfina, nas doses de $0,04 \mathrm{mg} / \mathrm{kg}$ e $0,4 \mathrm{mg} / \mathrm{kg}$ (IM), respectivamente. Após 15 minutos, foi alocado um cateter em veia cefálica e realizou-se a indução com propofol $4 \mathrm{mg} / \mathrm{kg}$ e midazolam $0,2 \mathrm{mg} / \mathrm{kg}$, seguida de manutenção anestésica com isofluorano. Posteriormente à instrumentação, procedeu-se à técnica de anestesia por tumescência com solução gelada composta por ringer lactato, lidocaína $2 \%$ sem vasoconstritor e adrenalina, em um volume total de $15 \mathrm{~mL} / \mathrm{kg}$. Em média, o tempo de duração do procedimento foi de $74 \pm 18$ minutos. O pico plasmático de lidocaína deu-se entre 30 e 60 minutos após a infiltração da solução. O resgate analgésico foi realizado após sete horas, aproximadamente, da infiltração. Pode-se concluir que a anestesia por tumescência com lidocaína deve ser considerada como constituinte do protocolo anestésico e analgésico de cadelas a serem submetidas à cirurgia de mastectomia, proporcionando estabilidade de parâmetros, segurança e recuperação pós-operatória de boa qualidade.
\end{abstract}

Palavras-chave: cães, anestesia balanceada, dor, neoplasias

\begin{abstract}
The present study investigated the benefits of tumescent anesthesia with lidocaine in dogs undergoing mastectomy, seeking the patients' comfort and their postoperative recovery. Seven animals, with different weight and breed, who had cancer in the region of mammary chain underwent mastectomy surgery. All animals received the same anesthetic protocol being used as the association between acepromazine and morphine doses of $0.04 \mathrm{mg} . \mathrm{kg}^{-1}$ and $0.4 \mathrm{mg} . \mathrm{kg}^{-1}$ (IM), respectively. After 15 minutes a catheter was placed in the cephalic vein and induction with propofol $4 \mathrm{mg} . \mathrm{kg}^{-1}$ and $0.2 \mathrm{mg} \cdot \mathrm{kg}^{-1}$ followed by maintenance with isoflurane anesthesia was done. After instrumentation, we proceeded to the tumescent anesthesia technique with ice-cold solution consisting of Ringer's lactate, lidocaine $2 \%$ without epinephrine and adrenaline in a total volume of $15 \mathrm{~mL} . \mathrm{kg}^{-1}$. The average duration of the procedure was $74 \pm 18$ minutes. The plasmatic peak of lidocaine was between 30 and 60 minutes after infiltration. The rescue analgesic was performed after approximately seven hours of infiltration. It can be concluded that the tumescent anesthesia with lidocaine should be considered as a constituent of anesthetic and analgesic protocol in dogs undergoing mastectomy surgery providing parameter stability, safety and good quality postoperative recovery.
\end{abstract}

Keywords: dogs, balanced anesthesia, pain, cancer

Recebido em 25 de setembro de 2012

Aceito em 13 de março de 2013

E-mail: cjxabimussi@hotmail.com 


\section{INTRODUÇÃO}

A utilização da anestesia infiltrativa pela técnica de tumescência tem sido crescente em medicina veterinária, uma vez que ela é segura e prática, podendo ser aplicada como protocolo anestésico adjuvante em mastectomia em cadelas, principalmente naquelas que apresentam algum fator de risco à anestesia geral (Carlson, 2005; Lopes e Almeida, 2008).

Seu uso isolado, entretanto, não é viável, sendo, na maioria das vezes, necessária a anestesia geral concomitante devido às limitações de colaboração por parte do animal e à intolerância em permanecer em decúbito e imóvel durante o ato cirúrgico. A associação de ambas as técnicas diminui de maneira significativa o requerimento do anestésico volátil, além de incrementar a analgesia pós-operatória imediata, podendo, desta maneira, ocupar um papel de destaque como protocolo anestésico complementar para a realização de cirurgias oncológicas cutâneas (Do e Kelley, 2007).

Vários aspectos têm sido considerados para o uso da anestesia infiltrativa por tumescência na realização de mastectomia em cadelas, destacando-se a maximização bioquímica do anestésico, a capacidade do anestésico utilizado em atingir a pele e o tecido subcutâneo local, a maior disponibilidade do anestésico no local de administração, a menor absorção sistêmica do fármaco e, consequentemente, a diminuição da toxicidade sistêmica, o aumento da dose limite, a expansão mecânica das camadas da pele (hidrodivulsão) e a elevação da pressão hidrostática local, o que reduz o sangramento trans e pós-operatório (Klein, 1995; Behroozan e Goldberg, 2005).

No homem, Klein (1988) relata que a qualidade da analgesia peri e pós-operatória produzida pela anestesia local por tumescência possibilita a realização do procedimento cirúrgico de lipoaspiração sem anestesia geral. Da mesma forma, Bussolin et al. (2003) e Agarwal (2004) afirmam que, quando houver alto risco em submeter o paciente à anestesia geral, a anestesia por tumescência pode ser uma alternativa, propiciando a realização de procedimentos como debridamento de feridas por queimadura, antes realizados sob anestesia geral associada à anestesia local infiltrativa.
Tais propriedades benéficas da anestesia por tumescência se devem aos constituintes da solução, normalmente composta por um anestésico local, um fármaco vasoconstritor, uma substância reguladora de $\mathrm{pH}$ e uma solução de infusão intravenosa estéril. Eventualmente, podese associar a essa técnica um anti-inflamatório esteroidal. É importante ressaltar que, embora a literatura descreva diferentes formulações para o preparo da solução, ainda não há consenso quanto à padronização da solução anestésica utilizada na técnica de anestesia por tumescência. Devido à baixa toxicidade, a lidocaína tem sido o anestésico local mais frequentemente utilizado na solução (Klein, 1999; Futema, 2005).

Sendo assim, o presente estudo tem por objetivo avaliar a segurança da anestesia por tumescência com lidocaína em cadelas submetidas à mastectomia, bem como mensurar as concentrações plasmáticas do fármaco anteriormente citado e o tempo de analgesia pósoperatória.

\section{MATERIAL E MÉTODOS}

Após aprovação pelo comitê de ética local (Ceea), sob o protocolo 2009-06265, o presente estudo foi conduzido nas dependências do Hospital Veterinário "Luiz Quintiliano de Oliveira" da Faculdade de Medicina Veterinária de Araçatuba - Unesp, utilizando-se sete cadelas, de raças variadas, com idade entre sete e 14 anos e peso médio de $10 \pm 6 \mathrm{~kg}$. Os animais foram selecionados pelo serviço de clínica cirúrgica de pequenos animais para a realização de mastectomia radical bilateral ou unilateral, excluindo-se aqueles que apresentassem as seguintes características: presença de metástase em outros órgãos (especialmente detecção de imagem pulmonar radiográfica sugestiva de metástase nesse órgão), animais com temperamento muito agitado, animais com alterações cardíacas ou sob tratamento de qualquer outra enfermidade que pudesse interferir nas variáveis do estudo. Foram, portanto, incluídas no estudo as cadelas que não apresentaram alterações no exame físico (FC, $f$, temperatura retal e avaliação da coloração das mucosas visíveis), bem como no hemograma completo, nos exames bioquímicos (ALT, FA) e sorológico para leishmaniose (sendo negativo para leishmaniose por tratar-se de região endêmica), e animais cujos proprietários 
concordassem com a utilização deles e assinassem o termo de consentimento esclarecido. Para a realização da cirurgia, os animais foram submetidos a jejum alimentar de 12 horas e hídrico de duas horas e, na sala de preparo anestésico, foram submetidos a uma nova avaliação física (FC, $f$, TPC, temperatura retal e pressões sistólica, diastólica e média). $\mathrm{Na}$ sequência, as cadelas receberam como medicação pré-anestésica (MPA) maleato de acepromazina $(0,04 \mathrm{mg} / \mathrm{kg})$ associada ao sulfato de morfina $(0,4 \mathrm{mg} / \mathrm{kg})$ pela via intramuscular. Decorridos 15 minutos, realizou-se a tricotomia da região a ser abordada cirurgicamente, bem como do local selecionado para a cateterização, e foi fornecido suporte volêmico. Após punção da veia cefálica e conexão de fluidoterapia com ringer com lactato na taxa de $10 \mathrm{~mL} / \mathrm{kg} / \mathrm{h}$, o animal foi encaminhado ao centro cirúrgico, onde se realizou a indução com propofol $(2,5 \mathrm{mg} / \mathrm{kg})$ e midazolam $(0,2 \mathrm{mg} / \mathrm{kg})$ seguida da intubação e, na sequência, deu-se início à manutenção anestésica com isofluorano, em concentração suficiente para manter o animal em plano anestésico cirúrgico, respeitando-se os conceitos estabelecidos por Guedel (Plano II/Estágio III).

Nesse momento, foram posicionados os eletrodos para registro do traçado eletrocardiográfico, aferição de pulso e mensuração da saturação de oxi-hemoglobina, aferição da temperatura esofágica e variáveis capnométricas.

Realizou-se cateterização da artéria podal transversa para a coleta de amostras de sangue para dosagem de anestésico local plasmático, bem como para a monitorização da pressão arterial pelo método invasivo, e cateterização venosa (jugular) para a coleta de amostras de sangue para posterior dosagem de cortisol.

Decorrido o período de preparo do animal, o tecido a ser removido cirurgicamente foi delimitado por meio de caneta especial para marcação em pele.

A fim de reduzir a formação de hematomas, utilizou-se uma cânula de Klein para fazer a infiltração da solução por se tratar de um instrumento com ponta romba, com oito orifícios na extremidade distal do canhão dela. Realizouse antissepsia com álcool iodado e iodopovidine tópico (PVPI) da região a ser infiltrada e, posteriormente, a abertura de um orifício de entrada com uma agulha hipodérmica 40x12. Por meio da cânula posicionada no espaço alvo, iniciou-se a administração da solução de tumescência em um volume fixo de infiltração de $15 \mathrm{~mL} / \mathrm{kg}$ para todos os animais, conectando-se uma seringa de $20 \mathrm{~mL}$ à extremidade da cânula. A solução foi composta por $210 \mathrm{~mL}$ de solução de ringer lactato, $40 \mathrm{~mL}$ de lidocaína $2 \%$ sem vasoconstritor e $0,5 \mathrm{~mL}$ de adrenalina, resultando em uma solução a $0,32 \%$, de acordo com o proposto por Futema (2005), sendo confeccionado um novo frasco com a mesma formulação, caso o volume ultrapassasse $250 \mathrm{~mL}$.

A solução a ser infiltrada foi preparada com solução de ringer em temperatura entre 8 e $12^{\circ} \mathrm{C}$, e o pH dela foi mensurado por meio de fitas de mensuração antes da infiltração e depois do momento da exérese do tumor por divulsão com tração manual.

As observações das variáveis (FC, $f$, PAM e temperatura retal) tiveram início imediatamente antes da aplicação da MPA (MB - momento basal), logo após a indução anestésica (MI), 30 minutos após a administração da solução de tumescência (M30), 40 minutos após a administração da solução de tumescência (M40), 60 minutos após a administração da solução de tumescência (M60), 80 minutos após a administração da solução de tumescência (M80) e 120 minutos após a solução de tumescência, sendo repetidas a cada 40 minutos até o final do procedimento cirúrgico. A partir do $\mathrm{MI}$ foram coletadas amostras de sangue $(3 \mathrm{~mL})$ por meio de cateterização da veia jugular direita nos momentos M30, MA (momento arrancamento exérese por divulsão com tração manual), M60, MFS (momento final da sutura) e MR (momento resgate analgésico), para realização da dosagem sérica de cortisol pelo método de quimioluminescência tipo "sanduíche". Após as coletas, o material era centrifugado, e o soro armazenado em temperatura de $-80^{\circ} \mathrm{C}$.

Para a realização de dosagem plasmática do anestésico local, utilizou-se a técnica de cromatografia líquida de alto desempenho (HPLC), cujo protocolo consistiu da coleta de $1,5 \mathrm{~mL}$ de sangue da artéria podal transversa em seringas com heparina de lítio nos momentos M30, MA, M60, após quatro horas da infiltração (coincidindo com o início da avaliação da 
analgesia) e, a partir de então, a cada duas horas de infiltração até 12 horas desta. Uma última amostra foi coletada decorridas 24 horas da anestesia por tumescência. $\mathrm{O}$ material foi centrifugado, e o plasma armazenado em freezer a $-80^{\circ} \mathrm{C}$ para posterior processamento, como descrito a seguir:

pesagem do padrão analítico de referência da droga que será dosada e/ou utilizada como padrão interno (PI/IS). No presente caso, foi o padrão de lidocaína (Nortec Química, Rio de Janeiro, Brasil). São realizadas duas pesagens do padrão (lidocaína - M1P1 e M1P2 e ropivacaína - M2P1 e M2P2), sendo que uma será utilizada para o preparo da curva de calibração e a outra para o preparo dos controles de qualidade e do limite inferior de quantificação (LIQ), de forma que os valores devem ser compatíveis quando cruzados. Com isso, evitam-se erros acumulados durante o processo;

preparo das soluções estoque diluindo os padrões em solvente apropriado, no caso metanol/água $1 / 1 \mathrm{v} / \mathrm{v}$, as quais serão mantidas refrigeradas durante todo o estudo;

preparo das soluções de trabalho a partir das soluções estoque. Apenas estas sairão da geladeira para serem realizados os experimentos diários.

A partir dessas soluções de trabalho, são feitas as diluições dos dois analitos até uma concentração de $1 \mu \mathrm{g} / \mathrm{mL}$, que serão utilizadas no experimento de infusão direta, para o ajuste dos parâmetros do espectrômetro de massas, de modo a obterem-se as melhores condições para detecção e dosagem do analito.

Após a infusão e a calibração do equipamento, são feitas as diluições da curva de calibração (M1P1), dos controles de qualidade e do LIQ (M1P2) de acordo com o range previsto na literatura para a dose e as condições utilizadas. É realizada também a diluição do padrão interno, partindo-se da solução de trabalho desse padrão (M2P1)

Dos pontos da curva, preparam-se um ponto intermediário e o LIQ para que se iniciem os trabalhos de cromatografia. São testadas colunas cromatográficas e fases móveis compatíveis com os analitos, de modo a se obter um cromatograma bem definido e sem interferentes.

Com o método cromatográfico pronto, sendo capaz de se identificar o LIQ de forma robusta e específica, iniciam-se os testes para extração do composto da matriz biológica que será dosado. São testadas extrações por precipitação e diferentes extrações líquido-líquido, a fim de que se utilize o processo que apresentar melhor recuperação e melhor precisão.

Com o processo de extração definido e o método cromatográfico montado, é realizada uma corrida de validação que consiste na curva de calibração de 10 pontos em duplicata, sendo dois plasmas brancos, dois plasmas com padrão interno e duplicatas de oito pontos de diferentes concentrações preparados a partir das diluições. São acrescentadas sete réplicas dos pontos: limite inferior de quantificação (LIQ), controle de qualidade baixo (CQB), controle de qualidade médio (CQM) e controle de qualidade alto (CQA), que cobrem toda a extensão da curva de calibração.

A validação é considerada aprovada se a curva possuir coeficiente de determinação mínimo $\left(\mathrm{r}^{2}\right)$ de 0,99 e os desvios de acurácia dos CQs estiverem dentro do limite de $\pm 15 \%$.

Com a validação aprovada e a utilização de um método confiável, realiza-se a dosagem das amostras em uma lista de amostras desconhecidas (LAD) que possui sua própria curva de calibração preparada no dia e seu conjunto de controles de qualidade que cubram toda a extensão da lista, sendo intercalados de 10 em 10 amostras.

As concentrações são calculadas pelas razões das áreas do analito pelas áreas do padrão interno, o que corrige eventuais variações da extração e supressões iônicas, dando confiabilidade ao método.

Após o término da cirurgia e o retorno da consciência do animal, a dor pós-operatória foi avaliada. Para tanto, foram utilizadas a escala de Melbourne (Firth e Haldane, 1997), sendo considerado positivo para dor um escore maior ou igual a seis, de um total máximo de 20 pontos, e uma escala intervalar (Morton et al., 2005), sendo considerado positivos valores maiores ou 
iguais a três, de um total máximo de 10 pontos. Uma terceira variável utilizada foi o retorno da sensibilidade cutânea, verificado por meio da aplicação dos filamentos de von Frey, em sua versão eletrônica, onde se utilizavam 15 filamentos de calibres variados, em ordem decrescente, equivalentes a, no máximo, 62 gramas de pressão. Caso o animal se apresentasse como positivo para os três métodos de avaliação, era realizado o resgate analgésico com $4 \mathrm{mg} / \mathrm{kg}$ de cloridrato de tramadol associado ao meloxican na dose de $0,15 \mathrm{mg} / \mathrm{kg}$, por via subcutânea.

Os dados foram submetidos ao teste de ShapiroWilk para atestar distribuição normal. Posteriormente, foram submetidos à análise de variância com medidas repetidas e comparação de médias por meio do teste de Tukey, sob 5\% de significância.

\section{RESULTADOS}

A média de idade dos animais foi de $11 \pm 2$ anos, e o peso médio foi de $10 \pm 6 \mathrm{~kg}$. Dos sete procedimentos, quatro foram de mastectomia unilateral e três de mastectomia bilateral. O tempo médio de procedimento cirúrgico foi de $74 \pm 18$ min. Por se tratar de um trabalho clínico, os dois cirurgiões responsáveis pelo serviço de clínica cirúrgica do hospital veterinário realizaram os procedimentos, utilizando a mesma técnica e intercalando entre si.

As variáveis transoperatórias, como frequência cardíaca (FC), frequência respiratória $(f)$ e pressão arterial média, não diferiram entre os animais (Fig. 1, 2 e 3) e demonstraram estabilidade de parâmetros e plano anestésico adequado ao procedimento.

A temperatura média de todos os momentos foi inferior à apresentada em MB basal, sendo de $34,2^{\circ} \mathrm{C}$ o menor valor encontrado (Fig. 4).

A concentração plasmática da lidocaína reduziuse na maioria dos animais após o momento do arrancamento (MA: exérese por divulsão com tração manual), com exceção de um animal (animal 6), que apresentou um aumento dos níveis plasmáticos até 240 minutos (Fig. 5).

O tempo médio de analgesia e de retorno da sensibilidade cutânea, e, portanto, o momento em que foi realizado o resgate analgésico, foi, em média, de $7 \pm 2 \mathrm{~h}$. Entretanto, individualmente, as escalas mostraram variações diversas quando comparadas entre si (Tab. 1), como se pode ver nos animais 2, 3, 4 e 5 .

Os valores de cortisol para cada animal estão ilustrados na Tab. 2.

\section{DISCUSSÃO}

No presente estudo, a temperatura corpórea dos animais se reduziu em todos os momentos em relação à temperatura basal, o que pode ser explicado não apenas pelo fato de o paciente estar sob anestesia geral, mas também de a solução estar em temperatura de $10^{\circ} \mathrm{C}$ aproximadamente, o que reduz o sangramento transoperatório e a absorção do anestésico local, indo de encontro a Butterwick et al. (1999) e a Hustad e Aitken (2006), que descrevem o uso de uma solução de tumescência aquecida entre 38$40^{\circ} \mathrm{C}$ com a finalidade de reduzir a perda de temperatura corpórea, bem como diminuir o desconforto no momento da infiltração.

Em relação à velocidade de infiltração, a utilização de seringa não permitiu a padronização de taxa fixa, o que poderia ser obtido com a utilização de bombas de infusão. Tal padronização é desejada, uma vez que a velocidade de infiltração está diretamente relacionada ao conforto do procedimento, observando-se maior desconforto do paciente submetido à infiltração rápida de grandes volumes quando acordado ou levemente sedado, o que requer sedação mais potente, como afirmam Hanke et al. (1997). Contudo, tal fato não se reveste de grande importância no caso de medicina veterinária, em que as mastectomias são realizadas, de preferência, com o animal sob anestesia geral, como realizado no presente estudo. 


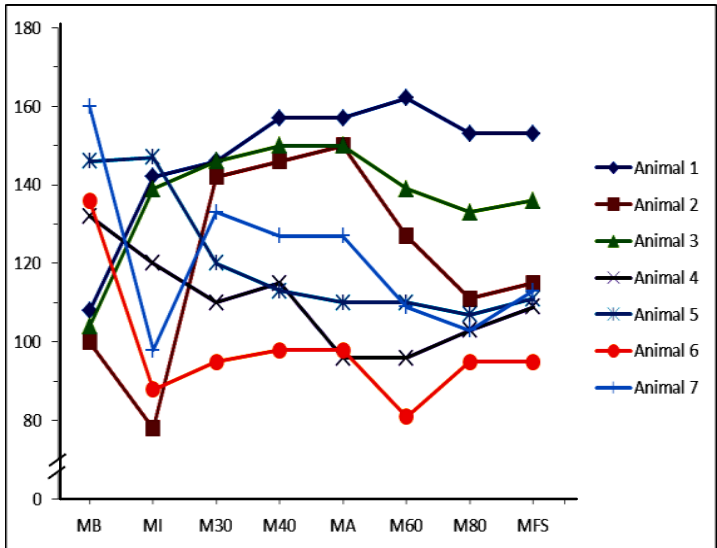

Figura 1. Valores da frequência cardíaca dos animais submetidos à técnica de anestesia local por tumescência (MA=momento exérese por divulsão com tração manual). Valores de referência: 60 a 160bpm (Feitosa, 2004).

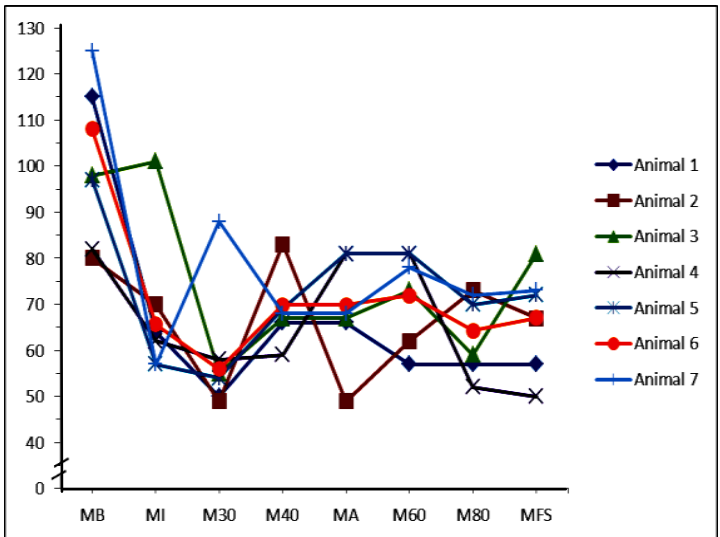

Figura 3. Valores da pressão arterial média dos animais submetidos à técnica de anestesia local por tumescência (MA=momento exérese por divulsão com tração manual). Valores de referência: $104 \pm 12 \mathrm{mmHg}$ (Haskins, 2007).

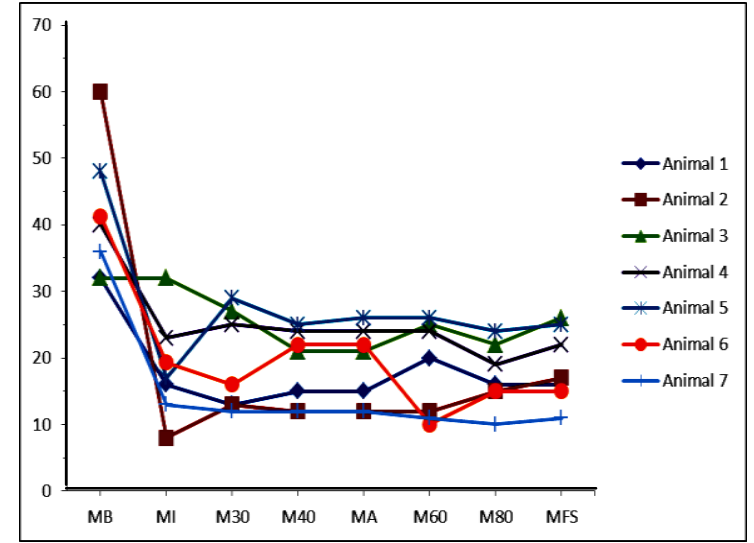

Figura 2. Valores da frequência respiratória dos animais submetidos à técnica de anestesia local por tumescência (MA=momento exérese por divulsão com tração manual). Valores de referência: 18 a 36mpm (Feitosa, 2004).

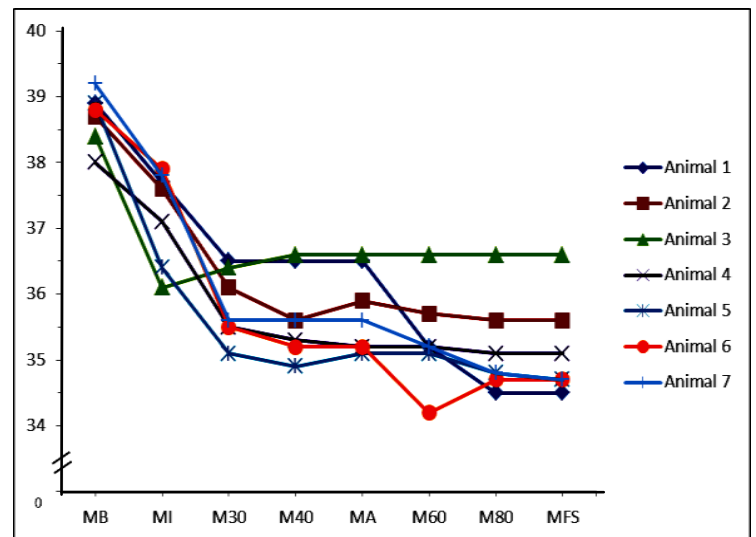

Figura 4. Valores de temperatura dos animais submetidos à técnica de anestesia local por tumescência (MA=momento exérese por divulsão com tração manual). Valores de referência: 37,5 a $39,2^{\circ} \mathrm{C}$ (Feitosa, 2004).

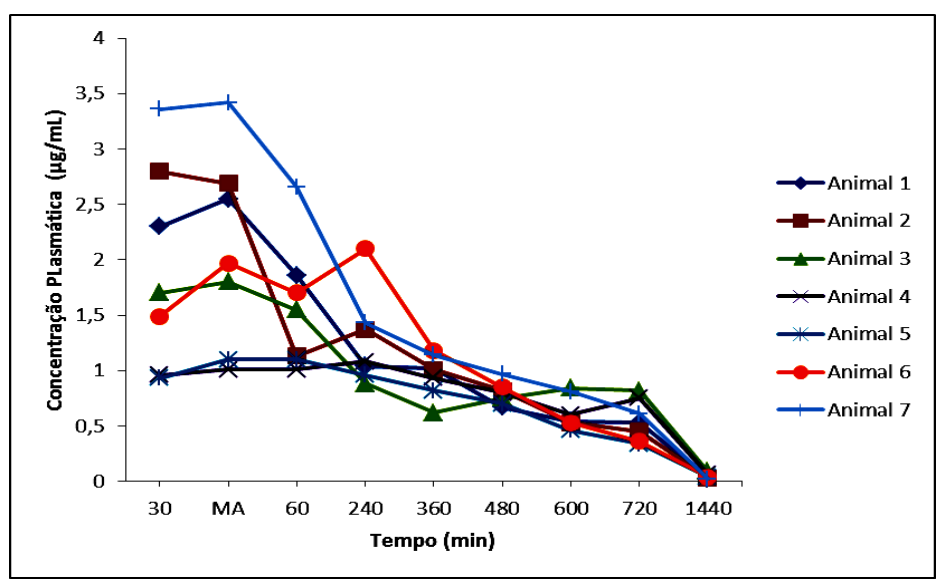

Figura 5. Concentração plasmática de lidocaína dos animais submetidos à técnica de anestesia local por tumescência (MA=momento exérese por divulsão com tração manual). 
Anestesia local por tumescência...

Tabela 1. Tempo, em horas, de resgate analgésico para cada animal de acordo com cada escala

\begin{tabular}{cccccccc}
\hline GL & Animal 1 & Animal 2 & Animal 3 & Animal 4 & Animal 5 & Animal 6 & Animal 7 \\
\hline Escala 1 & 10 & 4 & 5 & 4 & 4 & 4 & 4 \\
Escala 2 & 10 & 5 & 9 & 7 & 6 & 4 & 4 \\
\hline
\end{tabular}

Escala 1: Escala de Melbourne / Escala 2: Escala de Morton.

Tabela 2. Valores de cortisol sérico, em $\mu \mathrm{g} / \mathrm{dL}$, para cada animal em cada momento

\begin{tabular}{cccccccc} 
Momentos & Animal 1 & Animal 2 & Animal 3 & Animal 4 & Animal 5 & Animal 6 & Animal 7 \\
\hline M30 & 2,45 & 1,7 & 2,58 & 1,92 & 1,89 & 2,3 & 1,44 \\
MA & 1,94 & 1,98 & 1,27 & 5,48 & 2,53 & 2,18 & 1,46 \\
M60 & 1,06 & 1,2 & 1,82 & 5,48 & 2,53 & 4,97 & 2,84 \\
MFS & 4,63 & 0,69 & 0,57 & 4,24 & 2,07 & 5 & 3,54 \\
MR & 3,76 & 4,64 & 2,23 & 4,97 & 2,07 & 3,30 & 5,32 \\
\hline
\end{tabular}

MA=momento exérese por divulsão com tração manual; MFS=momento final da sutura; MR=momento resgate. Valores de referência: 0,5 a $6 \mu \mathrm{g} / \mathrm{dL}$ (Guimarães et al., 2007).

Ainda em relação à taxa de infusão, Butterwick et al. (1999) compararam a taxa de infiltração rápida com a lenta em 18 pacientes, a fim de mensurar a concentração sérica do anestésico local e a capacidade da adrenalina em induzir uma vasoconstricção de tal forma que impedisse a rápida absorção da lidocaína, e concluíram que não há correlação entre a taxa de infiltração e a concentração plasmática do anestésico local. No entanto, afirmaram que a concentração da solução deve estar entre 0,05 e $0,1 \%$, diferentemente do estudo em tela, em que ela foi de $0,32 \%$. Apesar disso, as concentrações plasmáticas de lidocaína variaram de 0,02 a $3,42 \mu \mathrm{g} / \mathrm{mL}$, ficando sempre abaixo das consideradas tóxicas e comportando-se de maneira semelhante ao valor relatado por Hustad e Aitken (2006), que encontraram $2 \mu \mathrm{g} / \mathrm{mL}$ como maior concentração plasmática de lidocaína dentro de um período de 36 horas, em dois pacientes submetidos à mesma técnica.

Rosaeg et al. (1998), ao utilizarem a escala visual analógica (EVA), observaram uma redução do escore de dor e do requerimento de analgésicos opioides em mulheres submetidas à redução de mama com a técnica de anestesia por tumescência com lidocaína por um período de 15 horas. Os dados aqui analisados demonstraram que o retorno da sensibilidade cutânea e a necessidade de resgate analgésico ocorreram, em média, sete horas após a realização da anestesia por tumescência, embora, individualmente, haja uma variação nos tempos de resgate das escalas, como pode ser visto na Tab. 1 (animais 2, 3, 4 e 5), o que poderia justificar a sensibilidade variável de cada método empregado. Esse período menor de analgesia pode ser explicado por diversos fatores, como a diferença de técnica cirúrgica e de sensibilidade das duas espécies, e, provavelmente também, pela utilização de métodos de avaliação diferentes entre os dois trabalhos. Como pode ser observado, o tempo de retorno da sensibilidade cutânea também se deu após sete horas da infiltração, o que pode ser avaliado com filamentos de von Frey, como descrito por Abimussi et al. (2011).

Adicionalmente, pode-se mencionar o fato de que o efeito mais duradouro em lipoescultura humana pode ser resultante da maior área infiltrada, com utilização de maior volume da solução. Por outro lado, o procedimento cirúrgico também pode ser um fator altamente importante, já que na lipoaspiração, ainda que parte da solução seja aspirada, resta uma fração residual considerável do anestésico, que continua a ser absorvida e a produzir efeito. Nas mastectomias em medicina veterinária, a técnica cirúrgica utilizada, que resulta em exérese por divulsão com tração manual de uma grande área tecidual, destaca, junto à área tumoral, a região contendo o gel formado pela solução infiltrada, o que, provavelmente, reduz de maneira significativa a quantidade de fármaco que continua a ser absorvido a partir de então. A transformação da solução líquida infiltrada em uma substância de consistência "gelatinosa" não se deve a nenhum constituinte da solução capaz de alterar sua forma física de líquido para gel. Sugere-se que tal fato possa ser atribuído à alteração do $\mathrm{pH}$ e à temperatura da solução, 
requerendo, contudo, maiores investigações a respeito.

Em relação ao cortisol, a média dos animais imediatamente após a indução anestésica (MI)

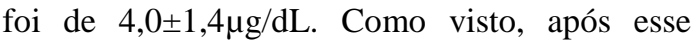
momento, os valores de cortisol reduziram em relação ao valor considerado basal (MI), o que pode ser atribuído aos efeitos de supressão da adrenal pela anestesia geral. Um dos animais, entretanto, apresentou valores de cortisol elevados $(5,48 \mu \mathrm{g} / \mathrm{dL})$ em relação ao valor basal (MI) no momento da exérese por divulsão com tração manual (MA), sendo este considerado o período de maior estímulo doloroso. As demais variáveis utilizadas para o reconhecimento clínico de qualquer sensação dolorosa (FC, $f$, PAM) não apresentaram alteração, o que sugere que esta seja em razão de um estresse alheio ao estímulo cirúrgico e justifica o fato de que, uma vez avaliado isoladamente, o cortisol é uma variável imprecisa como indicativo de dor.

Em relação às concentrações de cortisol no momento do resgate analgésico, todos os valores foram superiores aos valores obtidos na primeira aferição (MI), sendo o maior e o menor valor encontrado de 5,32 e $2,07 \mu \mathrm{g} / \mathrm{dL}$, respectivamente. Tal fato confirma os escores obtidos com as escalas de dor utilizadas no presente estudo em relação ao momento do resgate. Contudo, todos os valores encontrados estão dentro do intervalo de referência $(0,5$ $6 \mu \mathrm{g} / \mathrm{dL}$ ) de acordo com Guimarães et al. (2007).

\section{CONCLUSÕES}

Frente aos resultados apresentados no presente estudo, conclui-se que a anestesia por tumescência com lidocaína a $0,32 \%$ em cadelas submetidas à cirurgia de mastectomia é segura, resulta em concentrações séricas inferiores às consideradas tóxicas para a espécie e propicia analgesia pós-operatória imediata adequada, permitindo conforto na fase de recuperação anestésica.

\section{AGRADECIMENTOS}

À| Fundação de Amparo à Pesquisa do Estado de São Paulo - Fapesp e à Capes, pelo auxílio financeiro.

\section{REFERÊNCIAS}

ABIMUSSI, C.J.X; FONSECA, M.W.; FERREIRA, J.Z. et al. Utilização de filamentos de von Frey em cadelas submetidas à mastectomia - relato de caso. In: ENCONTRO DE ANESTESIOLOGIA VETERINÁRIA, 10., 2011, Campos do Jordão. Anais... Campos do Jordão: [s.n.] 2011. p.39. (Resumo).

AGARWAL, P. Safe method for release of severe post burn neck contracture under tumescent local anaesthesia and ketamine. Ind. J. Plastic. Surg., v.37, p.51-54, 2004.

BEHROOZAN, D.S.; GOLDBERG, L.H. Dermal tumescent local anesthesia in cutaneous surgery. J. Am. Acad. Dermatol., v.53, p.828$830,2005$.

BUSSOLIN, L.; BUSONI, P.; GIORGI, L. et al. Tumescent local anesthesia for the surgical treatment of burns and postburn sequelae in pediatric patients. Anesthesiology., v.99, p.13711375, 2003.

BUTTERWICK, K.J.; GOLDMAN, M.P.; SRIPRACHYA-ANUNT, S. Lidocaine levels during the first two hours of infiltration of dilute anesthetic solution for tumescent liposuction: rapid versus slow delivery. Dermatol. Surg., v.25, p.681-685, 1999.

CARLSON, G.W. Total mastectomy under local anesthesia: the tumescent technique. Breast. J., v.11, p.100-102, 2005.

DO, D.V.; KELLEY, L.C. Tumescent anesthesia: evolution and current uses. $A d v$. Dermatol., v.23, p.33-46, 2007.

FEITOSA, F.L.F. Exame Físico geral ou de rotina. In: FEITOSA, F.L.F. Seminiologia Veterinária A Arte do Diagnóstico. São Paulo: Rocca, 2004. p.77-102.

FIRTH, A.M.; HALDANE, S.L. Development of a scale to evaluate postoperative pain in dogs. $J$. Am. Vet. Med. Assoc., v.24, p.651-659, 1999.

FUTEMA, F. Anestesia por tumescência. In: ENCONTRO DE ANESTESIOLOGIA VETERINÁRIA, 7., 2005, São Luiz. Anais... São Luiz: [s.n.] 2005. p.88-97. (Resumo expandido). 
GUIMARÃES, S.M.; OLIVAO, V.N.L.S.; MAIA, C.A.A. et al. Correlação de diferentes períodos de jejum com níveis séricos de cortisol, glicemia plasmática, estado clínico e equilíbrio ácido-base em cães submetidos à anestesia geral inalatória. Braz. J. Vet. Res. Anim. Sci., v.44, p.96-102, 2007.

HANKE, C.W.; COLEMAN, W.P. III; LILLIS, J.P. et al. Infusion rates and levels of premedication in tumescent liposuction. Dermatol. Surg., v.23, p.1131-1134, 1997.

HASKINS, S.C. Monitoring anesthetized patients. In: TRANQUILLI, W.J.; THURMON, J.C.; GRIMM, K.A. Lumb \& Jones' veterinary anesthesia and analgesia. 4.ed., Ames: Blackwell, 2007. p.533-558.

HUSTAD, J.P.; AITKEN, M.E. Liposuction and tumescent surgery. Clin. Palstic. Surg., v.33, p.39-46, 2006.

KLEIN, J.A. Anesthesia for liposuction in dermatologic surgery. J. Dermatol. Surg. Oncol., v.14, p.1124-1132, 1988.
KLEIN, J.A. Tumescent technique chronicles: local anesthesia, liposuction and beyond. Dermatol. Surg., v.21, p.449-457, 1995.

KLEIN, J.A. Anesthetic formulation of tumescent solution. Dermatol. Clin., v.17, p.751759, 1999.

LOPES, B.C.C.; De ALMEIDA, R.M. Anestesia local no controle da dor: a técnica infiltrativa por tumescência - revisão de literatura. Clin. Vet., v.77, p.70-74, 2008.

MORTON, C.; REID, J.; SCOTT, E. et al. Application of a scaling model to establish and validate an interval level pain scale for assessment of acute pain in dogs. Am. J. Vet. Res., v.66, p.2154-2166, 2005.

ROSAEG, O.P.; BELL, M.; CICUTTI, N.J. et al. $B$. Pre-incision infiltration with lidocaine reduces pain and opioid consumption after reduction mammoplasty. Reg. Anesth. Pain. Med., v.23, p.575-579, 1998. 